\title{
Madrid, 1912. El reto del turismo
}

\author{
Ana Moreno Garrido \\ Universidad Nacional de Educación a Distancia (UNED). Guadalajara \\ amoreno@guadalajara.uned.es
}

Recibido: 07/06/2015

Aceptado: 08/09/2015

\section{RESUMEN}

El otoño de 1912 se inauguró el Hotel Palace en Madrid consolidando así la oferta de lujo en la capital. Al mismo tiempo, la ciudad, a través de la Asociación de Propaganda, organizaba un importante congreso de turismo que reunió durante una semana a todos los sindicatos del país. Ese mismo otoño, el director del Hotel Ritz hizo un interesante viaje internacional por Europa y América para promocionar su hotel y la ciudad. La coincidencia de factores no es casual y revela la importancia creciente que el turismo empezaba a tener para Madrid. Este artículo pretende, además, relacionar estos primeros pasos del Madrid turístico con otros aspectos del desarrollo del turismo nacional.

Palabras clave: Historia del turismo, Historia de Madrid, Hotelería de lujo, Congresos de turismo.

\section{Madrid 1912. Tourism as a Challenge}

\begin{abstract}
The Palace Hotel in Madrid was inaugurated in the autumn of 1912, consolidating the luxury hotel offerings in the capital. In parallel, the city, through the Asociación de Propaganda, was organizing an important tourism conference that for a week brought together all the syndicates in the country. That same month, the general director of the Ritz Hotel had an interesting international journey through Europe and America to promote his hotel and the city. The coincidence of the different factors was not accidental, and reveals the growing importance that tourism was starting to have for the city of Madrid. This article will also try to relate these first steps of Madrid tourism with other aspects in the development of national tourism.
\end{abstract}

Key words: Tourism History, Madrid history, Luxury Hotels, Congress of Tourism. 


\section{Introducción}

Hasta que, en octubre de 1910, no se inauguró el Hotel Ritz, Madrid no fue considerada una ciudad turística. Aunque la apertura del que en ese momento era el hotel más lujoso de España no garantizaba per se su incorporación a los grandes circuitos turísticos continentales, sin él, el gran turismo europeo ni siquiera consideraría la ciudad. La llegada del grand hotel no era sólo turismo, significaba, además, la aparición de una nueva forma de sociabilidad ya que sus salones empezarían a preparar a Madrid para los grandes negocios e importantes celebraciones. El Ritz no fue el único signo de los nuevos tiempos; en apenas dos años, el otoño de 1912, se inauguró otro hotel de lujo, el Palace que, con su gigantesco tamaño cuadruplicaba la capacidad hotelera de la ciudad. Ese mismo mes, la Asociación de Propaganda, organizaba un importante congreso de turismo que reunió durante una semana a todos los sindicatos de fomento del turismo nacionales y a sus homólogos franceses y portugueses para tratar los temas que más preocupaban al sector. La coincidencia en el tiempo de estos tres hechos no parece casual. Proyectada una gran reforma urbanística desde 1910 (la futura Gran Vía) la ciudad empezaba una transformación y modernización a la que no eran ajenas el turismo y sus posibilidades.

En este triángulo hubo, además, un nombre común: Luis Scatti ${ }^{1}$. El que fuera director del Hotel Ritz desde el verano de 1911 fue también vocal de la Asociación de Propaganda de Madrid y un testigo directo de cómo el recién llegado Palace en poco más de dos años se hacía con el control del Ritz dejando el gran lujo madrileño en manos de su propietario, George Marquet, uno de los hoteleros más importantes de Europa que, además, siguió ampliando el mercado español controlando otros hoteles en localidades de mucha importancia turística. Luis Scatti dejó el Ritz en junio de 1913, cuando cambio de dueño, pero antes de eso recibió el encargo de sus jefes de Londres de promocionar su hotel y, de paso, la ciudad. Se programó entonces un viaje que empezó sólo una semana después de clausurarse el congreso de turismo de Madrid y que le llevó durante dos meses, entre noviembre de 1912 y enero de 1913 a varias ciudades europeas y EEUU. Su cometido puede ser considerado como el primero de su especie, así como el primer contacto empresarial que se hizo para afianzar las relaciones turísticas entre España y Norteamérica. Su trayectoria profesional y su testimonio nos presentan una ciudad y un sector bastante más dinámicos de lo que pensamos, probablemente frenado por el estallido de la guerra del 14 .

\section{1910: el punto de partida. La cuestión del Gran Hotel}

El porqué el Madrid de principios de siglo era una ciudad extraña al turismo, y el turismo a ella, tiene que ver con lo poco interesante que resultó para los viajeros románticos que siempre prefirieron otros destinos muchos más sugerentes como Toledo,

1 Luis Scatti, nombrado ampliamente en este artículo y autor de un par de informes conservados en el Archivo General de la Administración (en adelante AGA) en el que se basa esta investigación está caracterizado con más profundidad en el punto 4 de este artículo. 
Sevilla o Granada. La deuda pendiente del turismo con la capital era consecuencia de su falta de atractivos y su oferta poco cosmopolita apenas limitada a una almendra central en torno al eje Puerta del Sol-Estación del Mediodía (luego conocida como Atocha) y un par de calles o paseos elegantes como la calle de Alcalá o el Salón del Prado. Aunque el Museo de Pintura tenía fama europea y pasar por la ciudad era prácticamente indispensable por el trazado radial de la red ferroviaria española que hacía de la ciudad un inevitable (y lento) nudo de comunicaciones, Madrid estaba muy lejos de figurar en el catálogo indispensable del gran turismo europeo ${ }^{2}$.

La necesidad de construir un gran hotel de turismo era una de las urgencias del Madrid turístico de principios de siglo. Los escasos establecimientos que se pudieran llamar "hoteles", el París y el de la Paz en la Puerta del Sol, el Roma en Caballero de Gracia o el Inglés en la calle Echegaray no estaban a la altura de lo que merecía una capital y el gran turismo ni siquiera los consideraba. Se cita 1902, año de la proclamación de Alfonso XIII, como fecha de inicio de la cuestión del "gran hotel" porque demostró la incapacidad de la ciudad para alojar visitantes y huéspedes de nivel, inconveniente que se hizo aún más evidente en 1906, año de la boda real, cuando los aristocráticos invitados tuvieron que ser alojados, a la vieja usanza, en casas y palacios nobiliarios. Fueron varios los interesados en construir el tan demandado gran hotel en Madrid. En 1902, con motivo de la proclamación de Alfonso XIII, el constructor catalán Joan Miró i Trepat se interesó por un solar en el paseo del Prado y llegó a encargar planos a Eduard Ferrés i Puig pero aquello no prosperó ${ }^{3}$. El siguiente plan, cuatro años después, tuvo mejor fortuna. Por dos fuentes distintas sabemos de esta iniciativa. Por un lado, la documentación conservada en el Archivo de Palacio a propósito de las inversiones de Alfonso XIII en el futuro Hotel Ritz menciona como antecedente unas negociaciones desde octubre de 1906 hasta mayo de 1907 "que no prosperaron" entre la Sociedad Colón (donde estaban los marqueses de Comillas y Urquijo) y el marqués de Guadalmina con la casa Warin de Londres ${ }^{4}$. Por otro lado, un informe de Luis Scatti menciona una iniciativa por parte de Jesús Carrasco, arquitecto en la Dirección General de Correos, cliente habitual del Hotel Gran Colón de Barcelona que le sugirió a su propietario intentar la aventura madrileña de un gran hotel en la calle Príncipe o Princesa, chaflán Carrera de San Jerónimo, frente a la calle Sevilla. Al encontrarse con el problema de la financiación pidieron al propio Scatti (en ese momento director del Gran Hotel Colón de Barcelona) que viajase a Londres

2 Sobre las transformaciones de ese Madrid de principios de siglo que aspiraba a ser metrópoli, consultar, BAHAMONDE MAGRO, Ángel y OTERO CARVAJAL, Luis Enrique (eds): La sociedad madrileña durante la Restauración: 1876-1931,Madrid, Consejería de Cultura de la Comunidad de Madrid, 1989; BAKER, Edward: Madrid Cosmopolita. La Gran Via, 1910-1936, Madrid, Marcial Pons, 2009; FERNÁNDEZ DE LOS RÍOS, Ángel: El futuro Madrid. Madrid, Imprenta de la Biblioteca Universal Económica, 1868; PINTO CRESPO, Virgilio (coord): Madrid, Atlas histórico de la ciudad, 1850-1939. Madrid, Fundación Caja de Madrid-Ludwerg Editores, 2001; RODRIGUEZ MARTIN, Nuria: La capital de un sueño: Madrid 1900-1936: La formación de una metrópoli europea (Tesis doctoral inédita. Madrid, UCM, 2013) y RUEDA LAFFOND, José Carlos: Madrid, 1900. Proyectos de reforma y debate sobre la ciudad; 1898-1914. (Tesis doctoral inédita. Madrid, UCM, 1993).

3 MONTOLIÚ, Pedro: “Casi 100 años de historia” en Palace Hotel Madrid, Lungwerg, 2008, p.30.

4 Administración general, Legajo 1166, expediente 4 inversiones reales en Ritz Archivo General de Palacio (en adelante AGP) 
para negociar en un momento que parecía inmejorable porque en 1906, fecha de la boda del rey, era más evidente que nunca la necesidad del gran hotel en Madrid. Según Scatti, en Londres una compañía constructora manifestó bastante interés, pensando en organizar un banco anglo-español para financiarlo pero fue el atentado de la boda de los reyes lo que alarmó a los inversores y dejó en suspenso la creación del hotel $^{5}$.

Aunque las fuentes no tienen conexión entre sí, debe de tratarse del mismo proyecto porque coincide el nombre, Colón, y coinciden los hipotéticos inversores: los ingleses. E incluso aparece ya la figura de Luis Scatti, que al viajar a Londres conoció a Harris, uno de los socios de la Ritz Development que años después le ofrecería el puesto de director del Ritz madrileño. Aunque las negociaciones no prosperaron inmediatamente, tampoco cayeron en saco roto porque las dos partes interesadas volvieron a retomar el contacto y

finalmente, en febrero de 1908 se iniciaron unas negociaciones que llegarían a buen puerto con la sociedad Ritz (Ritz Development) gestora de los Ritz de Londres y París y el Carlton de Londres. Los socios eran los señores Higgins y Harris y el barón Gunzburg. El director general era Mr. Elles y el director de Londres, el Sr. Amino. ${ }^{6}$

Ambas partes volvían a coincidir: un grupo promotor español, encabezado por los marqueses de Guadalmina y Urquijo, y la necesaria inversión inglesa que la puso la Ritz-Hotels Development Company.

El porqué contactar con Ritz tenía mucho que ver con los gustos y personalidad del Rey que, parece ser, era un entusiasta del parisino y porque garantizaría el nivel exigido para el hotel que se quería para Madrid. Desde la inauguración del primer Ritz de la Place Vêndome de la capital francesa en 1898, el gran lujo llegaba a la hotelería. El "estilo Ritz" basado en un proyecto integral (estética-higiene-eficacia) estaba destinado a lograr un ambiente elegante y acogedor que hiciera sentir al cliente como en su casa. Fue una revolución basada en el mantenimiento del lujo al que estaban acostumbrados sus huéspedes, pero también en la incorporación de un buen servicio de restauración asistido de nuevas normas y estilos en el comer, la eliminación de los cubiertos a precio fijo y la invención de la grande carte. Elevó tanto nivel que dignificó la profesión de la hotelería y la restauración gracias a la modernización e higiene de sus establecimientos que contaban con el mejor cocinero de su tiempo, Escoffier, así como una amplia gama de servicios a cualquier hora y una gran dotación de personal.

Ese tipo de lujo es el que llegaría a Madrid el otoño de 1910. En plenas negociaciones con los ingleses, en enero de 1908 se hizo la tira de cuerdas en el terreno elegido, un inmejorable solar en el Paseo del Prado, junto al museo de pintura que permitiría al hotel tener fachadas sobre el propio Salón del Prado, la Plaza de la Lealtad y la calle Felipe IV además de vistas sobre la iglesia de los Jerónimos, una de las más elegantes de Madrid. En los meses siguientes, se constituyó una comisión de

5 SCATTI, Luis, Notas referentes al desenvolvimiento de la hostelería de Madrid entre 1901-1913, AGA 03. 49. 02.12104

6 SCATTI, Luis, Notas referentes..... 
estudios $^{7}$ domiciliada en Madrid y, en julio, se creó la Sociedad Hotel Ritz S.A con un consejo de administración entre los que figuraban representantes de la Ritz-Hotels Development company, los citados Gunzburg, Harris y Higgins y, entre los españoles, Albiz, Errazu, Ivanrey, Urquijo y Güell, además de Mariano de Cuadra como secretario. Se pensó en un capital de cinco millones (mitad acciones, mitad obligaciones) pero finalmente fue algo más de la mitad dividida en 5.000 acciones de a 500 pesetas. En el costo estimado del hotel sobresalía la construcción, valorada en 2.500.000, seguida del 1.150.000 del terreno; 500.000 para mobiliario; 450.000 equipamiento y 400.000 pesetas de gastos ${ }^{8}$.

Para asumir el coste, la Sociedad Ritz tanteó al Banco Hipotecario, el Banco Hispanoamericano, Sres Arnús y Cía, E. Sainz e hijos y Urquijo y Cía ${ }^{9}$ aunque, finalmente giraría financieramente en torno a la órbita del Banco Urquijo y del Banco Español de Crédito. La Sociedad Hotel Ritz S.A se creó con un capital fijo de 3.725 .000 pesetas en 5.450 acciones ordinarias de 500 pesetas cada una y 2.000 acciones preferentes de 500 pesetas. Estas 450 acciones se entregaron en canje de 900 bonos sin mención de valor que fueron creados a su fundación a la Ritz-Hotels Development Company en pago de servicios y concursos. La mayor parte del capital fue suscrito por inversores españoles como el conde de Agrela, el Banco Urquijo y el vizconde de Güell. ${ }^{10}$ Un inversor especial fue la propia familia real que compró acciones de la primera emisión a nombre del Rey, 110 obligaciones por 50.600 pesetas, a nombre del príncipe de Asturias en la misma cantidad y 55 obligaciones (27.500 pesetas) la reina madre ${ }^{11}$. Sin embargo, según afirmaría Scatti después, el Ritz se montó con un capital

que resultó insuficiente. Se tenía que haber hecho una ampliación de capital de un millón más y no se hizo. Al inaugurarse se encontró con un excedente de gastos de 762.465 para el que no tenía ni reservas, ni fondo de caja. Pensaron que el hecho de implantarse un Ritz en Madrid bastaría, pero requería de una clientela de élite que, en realidad, no era tan amplia. El Banco Urquijo tenía que ser amortizado en tres meses lo que no ocurrió ${ }^{12}$.

El recién nacido hotel pronto tuvo problemas económicos que determinarían su suerte ya que George Marquet, el hotelero belga y su rival más directo, supo, desde el principio, de su debilidad financiera.

7 La comisión Landecho, Cuadra y Comyn estaba compuesta por Luis de Landecho y Antonio Comyn Crooke quienes, además de ser cuñados, ya habían sido socios en otros negocios como la construcción del ferrocarril entre Amorebieta y Guernica del que fue concesionario Landecho y donde también tuvo participación el marqués de Urquijo. Landecho, como arquitecto, firmaría los planos del Ritz de Madrid. Antonio Comyn, fiscal del Tribunal de Cuentas, senador y miembro del partido conservador fue asiduo del Ateneo y socio de la Sociedad Española de Excursionistas.

8 Administración general, Legajo 1166, expediente 4 inversiones reales en Ritz , ( AGP)

9 Administración general, Legajo 1166, expediente 4 inversiones reales en Ritz, (AGP)

10 GORTAZAR ECHEVARRIA, Guillermo: Alfonso XIII, hombre de negocios. Persistencia del Antiguo Régimen, modernización económica y crisis política (1902-1931), Madrid, Alianza Editorial, 1986, pp.80-81

11 Administración general, Legajo 1166, expediente 4 inversiones reales en Ritz, (AGP)

12 SCATTI, Luis, Notas referentes...... 
Pero, volviendo atrás en el tiempo y antes de todo eso, nada más constituida la sociedad, se presentó al ayuntamiento una Memoria "para la edificación de un hotel de nueva planta para viajeros en la Plaza de la Lealtad, 1" según los planos de Charles Mewes, arquitecto del Ritz parisino que garantizaba un establecimiento muy similar, y que estuvo firmada por los arquitectos españoles Luis de Landecho y Lorenzo Gallego $^{13}$. En piedra, ladrillo y hierro, el Ritz de Madrid era lo más parecido a su homólogo francés. Con sus ocho plantas, cinco de habitaciones, y capacidad para 200 huéspedes, tenía una sala de baile, varias salas de lectura y de reuniones y, sobre todo, y como gran novedad, un hermoso jardín de invierno con palmeras y plantas exóticas que, en invierno, se aclimataban gracias a un novedoso sistema de calefacción. Su primer director fue Antonio Mella que había trabajado en los Ritz de París y Londres y en el Esplanade de Hamburgo pero en escasos nueve meses, julio de 1911, sería sustituido por Luis Scatti.

$\mathrm{Su}$ inauguración fue todo un acontecimiento. A las 5 y media de la tarde del 2 de octubre de 1910, el presidente del gobierno, Canalejas, acompañado de cuatro ministros y miembros de la Ritz-Hotels Development Company esperaban al Rey y su familia que llegaban al son de la marcha real. La "gran obra patriótica" concluía con éxito y, por fin, Madrid tenía su gran hotel. Sin embargo, para algunos, como su futuro director, Luis Scatti, el hotel nacía amenazado de muerte por la inminente apertura del "mastodonte" del Palace ${ }^{14}$ que, además, se construyó justo enfrente. Cuando fue nombrado responsable del mismo expresó sus dudas, pero los inversores le tranquilizaron. Detrás del Ritz estaban el Rey y la Banca Urquijo ${ }^{15}$. A pesar de las palabras alentadoras, abrió el Palace en el otoño de 1912

y empezó la prevista lucha, no única y exclusivamente por los precios, sino porque el Ritz era mucho mejor así que toda la preocupación de George Marquet padre fue hacerse con él y lo consiguió con facilidad y con el mayor asombro por mi parte ${ }^{16}$.

Aunque, como dijo Scatti, Marquet maquinase desde el principio para hacerse con el más pequeño, pero más exclusivo Ritz, fue su mala situación económica lo que determinó su futuro. Una vez más, los papeles de Scatti son reveladores. La deuda que acumulaba el hotel desde su inauguración preocupaba a sus socios ingleses que "empezaban a estar fatigados de la poca consistencia financiera e indecisa de la Sociedad Ritz de Madrid”. Quizás fue la mezcla de la deuda, la presión de Marquet o las ilusiones desinfladas al coincidir en el tiempo dos hoteles de lujo, enfrente el uno del otro y, en el caso del Ritz, demasiado pequeño para poder competir, el caso es que, según Scatti, el Ritz se rindió sin poner resistencia. Pero antes de ponerse en manos del poderoso belga, el 30 de junio de 1913, la Sociedad Ritz aumentó su capital en un millón de pesetas por la creación de 2.000 acciones preferentes con $6 \%$ de interés que se ofrecieron a los accionistas en una proporción del 40\% de las que tenían en ese momento. También en ésta participó el Rey que puso otras 5.000 pesetas, un

\footnotetext{
13 Plano del Hotel Ritz .Archivo de la Villa 17-39-23

14 SCATTI, Luis, Notas referentes.....

15 SCATTI, Luis, Notas referentes.....

16 SCATTI, Luis, Notas referentes...
} 
$25 \%$ de 40 acciones preferentes $6 \%$ reservadas para él en la última emisión ${ }^{17}$. No fue suficiente. En agosto de 1913, Mariano de Cuadra, secretario del Consejo, escribía a Scatti para decirle que habían decidido vender a Marquet que se haría cargo de su explotación garantizando un rendimiento efectivo al capital social y que les pagaría un canon anual de 375.000 pesetas ${ }^{18}$. Scatti aporta datos económicos de la situación económica a 30 de junio de 1913 para señalar lo incomprensible del caso. Con pérdidas de 35.833,61 pesetas no parecía estar justificada la venta de un hotel que, a pesar de su corta vida, ya era un emblema de la ciudad. En cuanto Marquet se hizo con él, adoptó su sistema de explotación basado en el subarriendo de los servicios de comedor y cocina, fórmula inadmisible en los hoteles Ritz donde solo existía la dirección total única. Con la llegada del nuevo dueño, Scatti, dejaba el hotel.

\begin{tabular}{|l|r|}
\hline Coste de explotación & $305.665,02$ \\
\hline Intereses Banca Urquijo & $17.262,35$ \\
\hline Varios Sr. Cuadra & 450,00 \\
\hline Intereses obligación & $57.440,00$ \\
\hline Amortización & $22.500,00$ \\
\hline & $403.317,37$ \\
\hline Beneficio bruto & $367.483,76$ \\
\hline Pérdidas & $35.833,61$ \\
\hline
\end{tabular}

Fuente: Notas referentes al desenvolvimiento de la hostelería de Madrid entre 1901-1913 AGA, 03. 49. 02. 12104

Fig. 1. Balance de la cuentas de la Sociedad Hotel Ritz S.A a junio de 1913.

\section{Sólo dos años después: 1912, el Hotel Palace y el desembarco en España de George Marquet}

Muchas fuentes coinciden en señalar que en el origen de la historia del Hotel Palace de Madrid estuvo una conversación informal entre Alfonso XIII y el hotelero belga Georges Marquet en la playa de Deauville mientras el monarca asistía las carreras ${ }^{19}$. Según esas fuentes fue el rey el que propuso al hotelero que construyese un gran hotel en Madrid al estilo de los grandes hoteles europeos y, testigo de esa conversación y ese compromiso, aceptado por el hotelero, fue el marqués de la Vega-Inclán, recién nombrado comisario de turismo español ${ }^{20}$. La anécdota se ha repetido y se ha aceptado como válida pero cuesta creer que fuese precisamente ese año, cuando se creó la Comisaría Regia de Turismo, en el que se puso la primera piedra del hotel madrileño.

\footnotetext{
17 Fondo Alfonso XIII. Administración general, Legajo 1189, expediente 18. Ampliación de capital en 1913, (AGP)

18 SCATTI, Luis , Notas referentes... y GORTAZAR, Guillermo, Alfonso XIII.... p 80-81

19 GORTAZAR, Guillermo, Alfonso XIII.....p. 81 y Palace Hotel Madrid, p.80.

20 MONTOLIÚ, Pedro, "Casi cien años .......p. 32
} 
Por lo tanto, si Vega-Inclán fue testigo de esa conversación no era todavía comisario de turismo y si tuvo lugar fue, al menos un par de años antes de 1911. Sí sabemos que, en un momento dado, el comisario de turismo intervino como intermediario entre el Rey y el hotelero, aunque eso sería más adelante, ya para la inauguración del hotel, el otoño de 1912, durante los preparativos de la gran fiesta de inauguración. Otro detalle confirma que, en algún momento, el comisario de turismo se implicó y es que VegaInclán recibió el Cordón de la Corona del gobierno belga ${ }^{21}$.

George Marquet I (1886-1947) es un nombre indispensable del gran turismo europeo. Belga de nacimiento, llegó a poseer uno de los mayores grupos hoteleros continentales, la Compagnie International des Grands Hotels Européens, que incluía el Palace y el Astoria de Bruselas, el Claridge's de París, el Lugdunum Palace Hotel de Lyon, el Negresco de Niza y el Chateau d'Ardenne. Sus negocios eran, sobre todo, los hoteles pero también tuvo participación en la Compañía Internacional de Coches-Cama de su compatriota y otros intereses en la importante industria del ocio y turismo de la Belle Epoque, como casinos e hipódromos. Rival de César Ritz, su estilo hotelero estaba basado, como él, en el concepto del lujo (tuvo querencia por el nombre palace para sus hoteles, ya fueran nuevos o comprados) pero su estilo fue mucho más grandioso y audaz. Prueba de ello fue su apuesta por las grandes firmas de ingeniería, las únicas que le garantizaban poder trabajar con estructuras de hormigón armado, sus preferidas, el enorme tamaño de sus hoteles o poder ofrecer aquello que no tenía nadie como los baños individuales y el teléfono. Su reinado en la hotelería fue, además, más largo que el de Ritz, internado en un sanatorio desde 1913 y, en consecuencia apartado demasiado pronto de un negocio que quedaría en manos de la Ritz-Hotels Development Company.

Parece ser que Marquet tenía interés por España y Madrid desde principios de siglo y que llegó a hacer una sustanciosa oferta por el Ritz, ya en construcción, que fue denegada ${ }^{22}$. Descartada esa opción, pensó entonces en construir su propio hotel. En plena transformación urbana, la expansión más interesante de la ciudad giraba en torno a la Gran Vía, cuya primera piedra se puso en 1910 y que estaba llamada a convertirse en la calle más importante del nuevo Madrid pero finalmente se decantó por un solar con más solera. No hizo mala elección cuando se decidió por el inmenso terreno de 6.000 metros cuadrados, al otro lado de la plaza de Cánovas del Castillo, vacío desde que, en 1895, fuese demolido el antiguo palacio de los duques de Medinaceli. El emplazamiento era perfecto, quizás no tan elegante como el de su "rival", enclavado en el propio paseo pero resultaría fundamental en la historia del hotel porque al estar más próximo al congreso de los diputados, el Palace pronto pasaría a ser cita obligada de diputados, hombres de negocios y periodistas políticos, convertido, de alguna manera, en la prolongación más relajada de la vida política madrileña.

En el Archivo de la Villa se conservan los planos del hotel fechados en diciembre de 1910, otro argumento más para descartar la anécdota del triángulo Vega-Inclán-

21 MENENDEZ ROBLES, María Luisa: El marqués de la Vega-Inclán y los orígenes del turismo en España, Madrid, Ministerio de Industria, Turismo y Comercio, 2006, p.69

22 DOMÍNGUEZ UCETA, Enrique,“ La arquitectura del Palace Hotel de Madrid” en Palace Hotel Madrid, Lunwerg, 2008. pp 81 
Alfonso XIII-Marquet en $1911^{23}$, firmados por el arquitecto catalán Eduard Ferres i Puig y el estudio belga de León Monnoyer et Fils. El Palace, como dijo Scatti, nacía con vocación colosal. El considerable solar fue ocupado en su totalidad ya que el gigantesco edificio adoptó la forma trapezoidal del terreno para aprovechar al máximo el casi medio millón de metros cúbicos, dando como resultado una espléndida fachada de trescientos metros de ancho frente a la fuente de Neptuno. Todo lo que rodeaba al hotel era grande: sus ochocientas habitaciones, sus novedades técnicas que incorporaron baño y teléfono en cada habitación, su jardín de invierno con espectacular cúpula de hierro y vidrio Art Nouveau, sus 625 empleados o su estructura de hormigón armado, única en Madrid. El poderío de su dueño también quedó claro desde el principio. Se calcula que la inversión fue de quince millones de pesetas y que presionó para que fuese construido en un tiempo récord, 18 meses transcurridos entre la colocación de la primera piedra en marzo de 1911 y su finalización el 20 de septiembre de $1912^{24}$. En sus planes grandiosos Marquet no escatimó esfuerzos garantizando a sus clientes hasta 55 billares Brunswich, cervezas alemanas traídas especialmente para el hotel o dos orquestas de zíngaros que tocarían por la mañana y por la noche, e incluso proyectó un auditorio (Teatro Palace) para más de mil personas y un aparcamiento subterráneo que no se llegaron a construir ${ }^{25}$. Tal despliegue de medios es lo que ha hecho pensar a algunos que el hotel nació marcado por la rivalidad con el Ritz al que siempre quiso superar en todo ${ }^{26}$. Como ocurriera con éste, su apertura fue todo un acontecimiento. El 12 de octubre de 1912 quedaba oficialmente inaugurado aunque sin la presencia de la familia real que se excusó por encontrarse de luto. El hotel ya había recibido sus primeros clientes y organizado recepciones varias pero para aquel día histórico se enviaron más de cuatro mil invitaciones y la fiesta, donde los representantes belgas tuvieron un protagonismo especial, se completó con un concierto de música clásica y un impresionante bufé.

El éxito del Palace fue extraordinario. En su primer año de explotación alojó a 90.664 viajeros que fueron 168.000 los dos primeros años (frente a los 42.000 del Ritz en su primer año) y en 1920 llegó a repartir dividendos del 45\% en $1920^{27}$. Su éxito estuvo basado en una explotación muy inteligente donde se jugó con el lujo de un gran hotel pero también con algunos detalles modernos, propios ya del siglo XX. Su cervecería alemana (hoy desaparecida) era uno de los espacios más singulares del edificio, con su música americana y billares, un ambiente mucho más mundano y cosmopolita de lo que un gran hotel de lujo estaría dispuesto a asumir; en su restaurante había gran carta pero también precios fijos, se instaló una oficina de Viajes Cook en sus bajos y en la publicidad de 1913 se mencionaban "arreglos muy ventajosos para familias y estancias prolongadas" lo que lo alejaba diametralmente del exclusivo y elitista Ritz del que apenas separaban unos pocos cientos de metros y al que, Marquet, no dejaba de mirar.

23 Archivo de la villa 23-484-160

24 Palace Hotel Madrid, pp.33-34

25 Palace Hotel Madrid, pp.33-34

26 DOMÍNGUEZ UCETA, Enrique, "La arquitectura...." p.81

27 GORTAZAR, Guillermo, Alfonso XIII.... p. 81 
Finalmente, en el verano de 1913, Marquet se quedó con el Ritz lo que le convirtió en el dueño del gran lujo madrileño; pero no paró ahí. Su siguiente objetivo fue el Hotel Continental de San Sebastián. Este hotel, desaparecido en 1972, se había inaugurado en 1884 en el turístico, y caro, paseo de la Concha a imitación del recientemente inaugurado Continental de Biarritz de 1882. El Continental era el clásico hotel litoral que formó parte del ensanche de la ciudad, de hecho su arquitecto, José Goikoa fue el artífice del nuevo San Sebastián. Que el belga terminó controlando el hotel donostiarra lo sabemos porque, cuando en 1916 empezaron las negociaciones para construir un hotel de lujo en Santander, la presencia de Marquet en una localidad tan cercana, y tan rival, como la guipuzcoana daba un cierto miedo a los santanderinos.

La idea de construir un gran hotel en Santander venía de lejos pero se concretó el 26 de febrero de 1916 cuando se constituyó la sociedad "para la construcción y explotación del Real de Santander". Como en el madrileño Ritz, también ahí estuvo el Rey que suscribió sólo cien acciones (de las 3.500 de la Sociedad) por 50.000 pesetas, dando así apoyo moral a una iniciativa local que tendría gran importancia para la ciudad ${ }^{28}$. Y también como ocurriera con el Ritz, parece ser que Marquet enseguida se interesó por él porque escribió a la junta de accionistas para arrendarlo. A finales de 1916 llegó a la ciudad cántabra. Cuatro días antes, una comisión del hotel en construcción donde estaban Botín como presidente, José Pardo, como secretario y el arquitecto del hotel, Javier González de Riancho, habían viajado a Madrid para cerrar el trato con el hotelero belga. La llegada de Marquet

aliviaba el temor de falta de experiencia ante una aventura empresarial de tamaña envergadura que se agravaba aún más por la competencia que, desde San Sebastián, ejercería el mago de la industria hotelera europea. ${ }^{29}$

La prensa local pareció satisfecha porque "en Santander no se saben hacer estas cosas. El Hotel Real tendrá que cerrarse. En San Sebastián está Monsieur Marquet, aquí, ¿a quién tenemos?" El 12 de julio de 1917, en plena temporada estival, se inauguraba ya con el sello Marquet: todas las habitaciones disponían de baño privado, las estructuras de hierro y madera fueron sustituidas por hormigón armado y en la ceremonia inaugural tocó su tradicional orquesta de zíngaros. Dos días después se abrió la segunda parte del casino, y, el 2 de septiembre, el hipódromo de Bellavista que también pasaron a estar controlados por el hotelero belga ${ }^{30}$

Terminada la guerra en Europa, Marquet seguiría interesado en ampliar negocio en una España cada vez más floreciente desde un punto de vista turístico. El 15 de marzo de 1920 se hizo con la explotación del Hotel de París de la Puerta del Sol que si bien no podía rivalizar con sus otras posesiones en la capital (Ritz y Palace) era un hotel interesante que a principios de siglo había representado la hotelería "moderna" en la ciudad y que, para otro tipo de clientes, seguía teniendo un importante poder

28 GORTAZAR, Guillermo, Alfonso XIII......p. 83

29 La Atalaya, 13 de julio de 1917

30 SAN EMETERIO GOMEZ, José: Hotel Real: recuerdos para un aniversario 1917-1992, Santander, Librería Estudio, 1992, pp. 88-89 y FLORES-GISBERT, Juan Carlos: Hotel Real de Santander, 1917, Santander, Tantin, 2012. 
de captación al estar ubicado en una zona magnífica de la capital, no tan exclusiva pero sí bulliciosa y comercial ${ }^{31}$. En 1929 fue el recién inaugurado Hotel Alfonso XIII de Sevilla, un lujoso establecimiento construido ex profeso para una ciudad volcada de lleno en la celebración de una exposición internacional. El sevillano fue la última inversión del belga que, por otra parte, dejaba el Real esa primavera. Aunque los propietarios rescindieron el convenio con Marquet en abril del 29 (lo que les obligó a pedir al Patronato Nacional de Turismo una subvención del 1\% durante cinco años que fue denegada ${ }^{32}$ ) de cara a la publicidad de ese año tan señalado desde un punto de vista turístico, la Compañía Internacional de Grandes Hoteles pudo venderse como el gran grupo hotelero del lujo español.

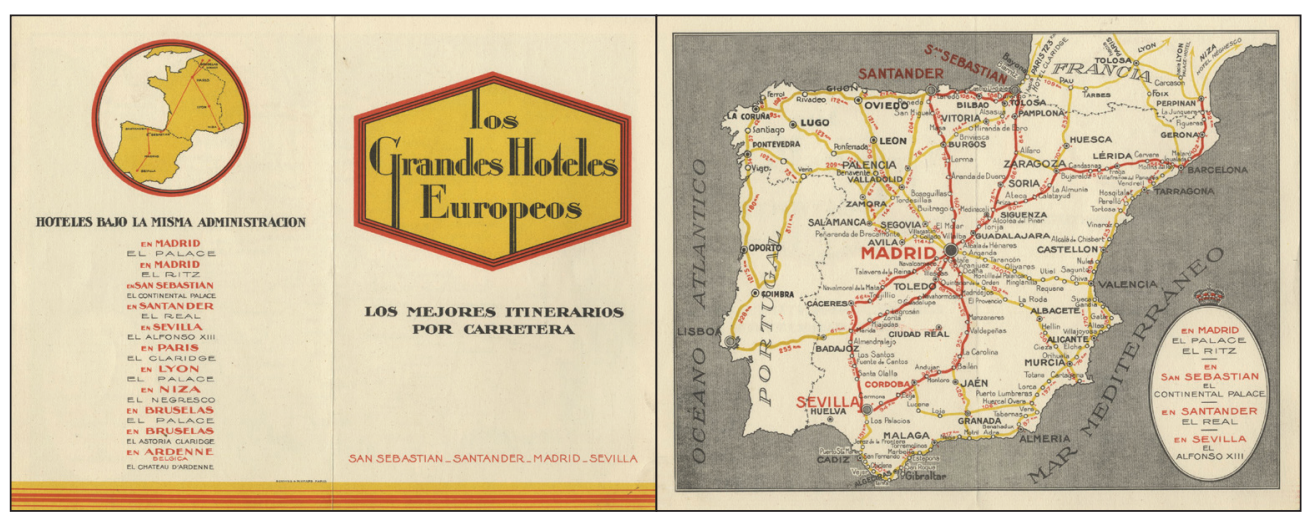

Fig. 2. Folleto publicitario Grandes Hoteles, Anverso y reverso.

\section{EI V Congreso Internacional de Turismo, la Asociación de Propaganda de Madrid e Hilario Crespo}

Sin embargo, la llegada del gran lujo a la ciudad no fue el único síntoma de la importancia creciente del turismo en Madrid. Como capital en crecimiento y por influencia directa del higienismo, los madrileños se aficionaron pronto al paseo por los entornos monumentales de la ciudad pero, sobre todo, fueron pioneros en establecer una renovada relación con su entorno. De esa manera la sierra madrileña es uno de los espacios nacionales más interesantes para medir el tránsito de las prácticas decimonónicas a las ya turísticas del nuevo siglo XX y laboratorio de excursionistas y aficionados a los deportes. Sin embargo, de todas las novedades en el tránsito del siglo que desembocarán en el turismo del siglo XX hay dos fundamentales: el velocipedismo y el asociacionismo. Ambas son las bases del turismo moderno en la ciudad.

Al principio el turismo fue deporte y los velocipedistas, turistas sobre dos ruedas. La importancia del velocipedismo en el origen del turismo es incuestionable y en

\footnotetext{
31 Informe al Consejo de Administración del ejercicio 1919-1920. Archivo del Hotel Palace de Madrid

32 03.49.02.11981 (AGA)
} 
Madrid, además, tuvo un hilo conductor muy claro en la persona de Hilario Crespo, uno de los nombres clave en la organización del turismo madrileño en la primera década del siglo XX. Crespo empezó siendo precisamente eso, un velocipedista más que, a principios de siglo, se convirtió en pequeño empresario junto a su hermano, abriendo una tienda de bicicletas y accesorios en la calle Arenal, 27. Pronto se debió comprar un coche porque en 1908, cuando se creó la Cámara Sindical Automovilística, su primer presidente fue él. Ese mismo año se fundó la Asociación de Propaganda de Madrid, el sindicato de iniciativas turísticas de la ciudad (que también tuvo su sede en Arenal, 27) y, en 1912, ya era el secretario del V Congreso de Turismo de Madrid que reunió a los profesionales más importantes de sector invitados por la asociación madrileña. La secuencia en la vida de Crespo es muy indicativa. De velocipedista a dueño de un automóvil y, de ahí, a implicarse en el naciente mundo turístico, un mundo en el que coincidió con algún poderoso porque el conde de Peñalver, introductor del automóvil en España en 1898, y al que conoció en la cámara sindical, estuvo en el comité organizador del congreso madrileño. Crespo, además, llegó a ser concejal del Ayuntamiento de Madrid y escribió sobre fomento de turismo. Es, sin duda, un representante clásico del grupo regeneracionista que, desde muy finales del XIX y, sobre todo, principios del XX se interesaron por el turismo convencidos de que, de desarrollarse al nivel de otras naciones europeas, traería importantes ventajas económicas, sociales e incluso políticas a una España que necesitaba con urgencia dejar atrás la grave crisis con la que terminó el siglo ${ }^{33}$. Aunque no fueron pocos, los más conocidos (e influyentes) fueron catalanes y mallorquines donde el turismo tuvo un discurso más sólido y la preocupación por su desarrollo fue mayor.

El congreso de Madrid no fue el único, pero sí el más importante de los congresos históricos de turismo celebrados antes de la guerra del 14, sobre todo porque fue el último y, en consecuencia, resume toda la trayectoria y madurez a la que había llegado el fenómeno y permite, por comparación con los otros, ver la evolución del sector. El primero de ellos se había celebrado en Zaragoza en 1908 en un contexto típicamente regeneracionista: una gran exposición hispano-francesa celebrada a propósito del centenario de los Sitios de 1808 pero que, en realidad, fue un homenaje y un culto al trabajo y una clara llamada a la renovación nacional. El organizador de la exposición fue Basilio Paraíso, un hombre del círculo costiano que encajó perfectamente la oportunidad de un congreso dedicado al turismo, una joven pero prometedora industria, en el contexto más amplio de la exposición zaragozana ${ }^{34}$. El sindicato de Zaragoza se acababa de crear y fueron las regiones limítrofes con Francia las primeras en absorber las novedades que llegaban del otro lado de los Pirineos. Una de estas fueron los sindicatos de iniciativa (en algunas localidades llamados asociaciones de propaganda) una modalidad de asociacionismo local importada de Francia (Grenoble, 1888) y que

33 Sobre los orígenes regeneracionistas del primer turismo español véase, MORENO GARRIDO, Ana: Turismo y nación. La definición de la identidad nacional a través de los símbolos turísticos. España, 19081929. (Tesis doctoral inédita. Madrid, UCM, 2004); GONZALEZ MORALES, Juan Carlos: Turismo en España 1905-1931. (Tesis doctoral inédita. Madrid, Universidad Carlos III de Madrid, 2003) y MORENO GARRIDO, Ana: Historia del turismo en España en el siglo XX, Madrid, Síntesis, 2007.

34 Conclusiones del I Congreso Internacional de turismo en Edición Oficial del Libro de Oro de la Exposición Hispano-francesa de 1908, Zaragoza, Imprenta del Heraldo de Aragón, pp. 354-358. 
resultó clave en el desarrollo del primer turismo europeo. Los sindicatos lo fueron casi todo al principio: oficinas de información turística, pequeñas agrupaciones locales de profesionales y, en un momento dado, lo que hoy llamaríamos lobbys de presión que conseguirían incluso presentar propuestas de ley o debates en el congreso. Y consiguieron algo que los gobiernos todavía no estaban en disposición de hacer: una unión nacional de los mismos y un intercambio internacional. Ese fue en realidad la gran aportación de los congresos: la superación de lo estrictamente nacional para internacionalizar los problemas y necesidades de un sector que es, por su propia naturaleza, internacional. Como todavía el turismo era una actividad relativamente regional los países que interesaron a los sindicatos españoles fueron, lógicamente, nuestro vecinos fronterizos: Francia y Portugal. La unión de los sindicatos de los tres países es lo que dio origen a la celebración de cinco congresos hasta 1914 cuando la guerra europea dio al traste con todos los buenos deseos de internacionalismo y hermandad que habían caracterizado las relaciones turísticas de la década de los diez.

Tras la celebración, bastante exitosa, del I Congreso en Zaragoza, hubo otras tres convocatorias más en San Sebastián (1909), Toulouse (1910) y Lisboa (1911) que prepararon el camino a Madrid. La decisión de celebrar un congreso en la capital se tomó justo los últimos días del congreso lisboeta del 11, probablemente gracias a los buenos oficios de Hilario Crespo que acudió a Lisboa como vicepresidente de la Asociación de Propaganda de Madrid y que trabajó duro para conseguir traer una nueva convocatoria, esta vez, española. Dos novedades en materia de turismo pudo plantear terminando el congreso del $11^{35}$. Por un lado, la creación en España, de un organismo dedicado al turismo, la Comisaría Regia de Turismo y de la Cultura Artística, que se hizo oficial en junio y la construcción, por fin, de un gran hotel de turismo en la ciudad, el Ritz, inaugurado el otoño anterior. Si ambas noticias se podían interpretar como síntoma de cambios, en realidad solo la presencia del Ritz tuvo un efecto directo sobre el congreso porque se aprovecharon sus salones para el banquete de despedida y se contó con algún representante dentro del comité organizador, pero el flamante comisario de turismo, Benigno de la Vega-Inclán, se excusó con un viaje a Nueva York "en cumplimiento de la alta y honrosa misión que me ha sido encomendada a favor de los intereses del turismo y de la cultura nacional" 36 para no asistir a un congreso en el que, en realidad, no estaba en absoluto interesado ${ }^{37}$. El divorcio pues entre el turismo oficial y el turismo real, el de base, se hizo evidente pues, desde muy pronto.

\footnotetext{
35 Memoire du IV Congrès Internationale de Tourisme (Compte Rendu Géneral), Lisbonne, 12-19 mai 1911

36 Memoria General del V Congreso Internacional de turismo de la Federación de los Sindicatos de Iniciativas Franco-hispano-portugués organizado por la Asociación de Propaganda de Madrid, Madrid, 24 al 30 de octubre de 1912, p. 60

37 Fondo Alfonso XIII. Turismo. Caja 15.592 (AGP)
} 


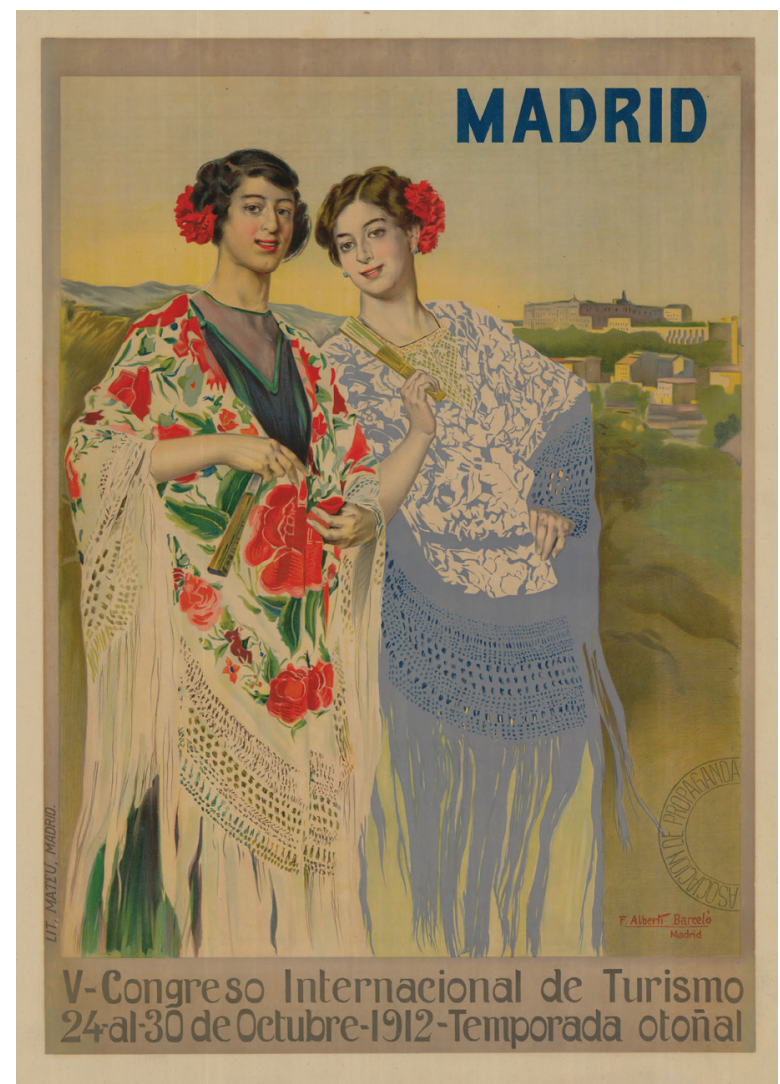

Fig. 3. Fuente: Colección de carteles del Centro de Documentación Turística de España, Instituto de Estudios Turísticos.

El congreso partía de "la importancia extraordinaria" que tenía para España cuanto pudiera contribuir al fomento del turismo internacional y para Madrid el poder medirse y rivalizar con las otras ciudades que habían acogido las otras convocatorias e, incluso, con los representantes de Francia y Portuga ${ }^{38}$. Se celebró entre el 24 y el 30 de octubre, una fecha que no parecía casual, ya que el otoño era la temporada turística por excelencia en Madrid "época que por su temperatura agradable rivaliza con la primavera" y que se pretendía que marcase "una nueva corriente de turismo" 39 que, de momento, empezaba con la llegada de los congresistas de los tres países. Para el congreso no se escatimaron esfuerzos: la sesión de apertura fue en el Paraninfo de la Universidad Central la mañana del día 24, presidiendo el ministro de Fomento Miguel Villanueva y el alcalde de Madrid, se contó con la presidenta de honor de Alfonso XIII y del presidente del gobierno, Canalejas. La clausura fue el 30 en ese

\footnotetext{
38 Memoria General del V Congreso Internacional... p. 39

39 Memoria General del V Congreso Internacional... p. 21
} 
mismo Paraninfo para, a continuación, trasladarse al Ritz a celebrar un banquete de despedida.

El ritmo y organización del congreso no difirió mucho de los cuatro anteriores: actos protocolarios, sesiones de trabajo y un programa completo de actividades y excursiones. La organización sorprende por su modernidad y el buen tono que rodeó al congreso pero, sobre todo, por la cantidad de tiempo del que disponían los congresistas, tanto nacionales como extranjeros, algunos de los cuales pasaron cerca de un mes en España. Primero, una recepción en el ayuntamiento la tarde anterior a la inauguración, luego el acto de apertura en la universidad, teatro, excursiones a Toledo, Segovia y El Escorial además de una excursión final a Andalucía, una exposición del Club Alpino Español en el Retiro, cuatro conciertos de la Asociación Wagneriana de Madrid 26, 28, 29 y 30 por la orquesta sinfónica de Madrid, corrida de toros, visita a las reales caballerizas y banquete final en el Ritz. Algunos de los congresistas extranjeros ya habían empezado el 1 de octubre a conocer España con un itinerario que empezaba en Barcelona bajaba hasta Andalucía y volvía a Madrid y remataron el mes con la participación en el congreso del día 24.

Las secciones y temas estuvieron en la misma línea que las de los congresos anteriores y eran, en definitiva, las preocupaciones reales del sector: comunicaciones, publicidad, el papel de las sociedades turísticas, arquitectura y hoteles. Algunos de los temas eran más nacionales pero otros necesitaban de la coordinación entre los sindicatos de los tres países fronterizos. Eran asuntos imprescindibles como la necesidad de establecer tarifas internacionales de excursiones o la exención de derechos de aduanas e impuestos municipales para promoción y carteles y así poder publicitar los destinos en otros países, o, por supuesto, la simplificación de formalidades en las fronteras. En lo referido al mercado español lo que más preocupaba al turismo nacional eran sobre todo el transporte y el alojamiento. Dos urgencias que seguían lastrando a la industria de los viajes. En este sentido se insistía en la necesidad de crear billetes de ida y vuelta y mejorar los billetes kilométricos (los preferidos de turistas y viajeros) pero también en el proyecto para la constitución de una cámara oficial hostelera que mejorase y luchase por la calidad de la hotelería nacional. Otros temas eran más novedosos y no aparecieron en el congreso zaragozano de 1908 como una memoria sobre los tres circuitos turísticos recomendados por el Real Automóvil Club (sorprendente porque todavía el parque automovilístico español era de poco más de cinco mil autos ${ }^{40}$ pero germen de lo que luego sería el Circuito de Firmes Especiales) o la atención destacada a temas que hoy calificaríamos de turismo urbano o cultural como las características que deben tener los edificios destinados al turismo, si moderno o tradicional, las propuestas de mejoras urbanas con incidencia directa en turismo o una interesante conferencia de Vicente Lampérez donde organizaba la geografía monumental española de acuerdo a hipotéticos itinerarios de turismo. Otros asuntos eran, realmente inclasificables y sorprendentes como la relación entre el turismo y el árbol o el turismo y el esperanto. ${ }^{41}$

40 Datos citados en "El progreso del automovilismo en España" en España Automóvil. Órgano oficial de la Cámara Sindical española de automovilismo (1907-1928), 1912.

41 Memoria General del V Congreso Internacional... 
Muy interesantes para tomarle el pulso al turismo de la época y originales en algunas de las propuestas y preocupaciones pero poco efectivos. Los congresos a duras penas pasaron de ser llamadas de atención a los poderes públicos para que se implicasen en el sector pero la lógica de los tiempos no les acompañaban, tendrían que pasar más de diez años y una gran guerra para que los Estados se tomaran en serio el turismo y viesen en él uno de los negocios más prósperos y prometedores de los años de entreguerras. Aunque quedaron emplazados para una siguiente convocatoria, con el congreso de Madrid acabaría el ciclo de congresos de sindicatos turísticos; el delegado de la oficina nacional de turismo de Portugal dijo en un momento que

ahora que del Oriente de Europa llega a nosotros el eco de los cañonazos, ahora que los odios de raza y religión encienden y avivan lavas de incendios destructores, aquí las únicas llamas que percibimos son las de los fuegos de alegría.... ${ }^{42}$

pero el estallido de la guerra en Europa un año y medio después se llevó toda una época histórica de turismo.

La relativa poca efectividad de los congresos turísticos no debe distraernos del asunto importante y es la atención creciente del turismo en la ciudad en un doble movimiento: el gran lujo, pero también el turismo de base. La financiación del mismo es indicativa de cómo tanto el comercio como pequeños profesionales o sociedad civil juzgaron necesario y provechoso la celebración del congreso madrileño. Un congreso de características similares estaba presupuestado en 125.000 pesetas, pero éste rondó las 150.000 que se consiguieron gracias a donativos de particulares y sociedades ya que el Ayuntamiento de Madrid solamente aportó 25.000 pesetas $^{43}$. Todavía en 1916 el presupuesto municipal para el fomento del turismo era de 7.000 pesetas que, parece ser, no siempre se destinaban a "los fines y propósitos consignados" 44

Hilario Crespo también fue vértice de otro asunto tan interesante como poco investigado: las relaciones entre el americanismo y el turismo, un tema que tendría consecuencias importantes en la administración turística nacional ya en los años 20 pero que empezó a gestarse en la primera década del siglo. El objetivo era "procurar que los millares de turistas de Sudamérica que vienen a Europa todos los años pasen por España, aunque sólo sea de tránsito" ${ }^{45}$ y hacer del turismo otro espacio común de relaciones entre España y los países americanos. En realidad, toda esta primera etapa de acercamiento al turismo latinoamericano era consecuencia de un movimiento más amplio surgido en el cambio de siglo para recuperar la presencia española en la América del sur. La primera asociación americanista, la Unión Iberoamericana que se había constituido en 1885 para estrechar lazos académicos con la América española (surgió en la Universidad de Madrid para habilitar títulos profesionales) fue el ger-

\footnotetext{
42 Memoria General del V Congreso Internacional ... p. 53

43 CRESPO GALLEGO, Hilario: Proyecto de Congreso Hispanoamericano presentado a la Cámara oficial de Comercio de la provincia de Madrid, 1914, p.10

44 CRESPO GALLEGO, Hilario: Fomento del turismo madrileño. Proposición presentada a la Excelentísima Corporación, 1915, p.27

45 Pro-Patria Número extraordinario de la revista cultura Hispano-americana dedicado al turismo, Madrid, Establecimiento tipográfico el Liberal, 1913, p. 348
} 
men de todo un movimiento que, más allá de lo cultural, trató de reconstituir las redes sociales y económicas con las burguesías trasatlánticas, y constituyó un influyente espacio de acción industrial, mercantil y empresarial español hasta la guerra de 1936. Aunque sería el americanismo empresarial catalán el que influyese, y mucho, en la primera administración turística española de 1928, ya el primer ambiente turístico madrileño representado en Crespo fue consciente de la importancia de la captación de americanos para el futuro turístico nacional. Crespo llegó a proponer hasta en dos ocasiones celebrar congresos de turismo hispano-americano (en los años 20 seguía insistiendo mucho en convocar uno paralelo a la exposición sevillana de 1929) y siempre se adjudicó la idea de la celebración de una fiesta-efeméride que conmemorase el descubrimiento de América, la que luego se llamó "fiesta de la raza" fijada para el 12 de octubre ${ }^{46}$.

\section{Hay que promocionar la ciudad. El viaje de Luis Scatti}

El 24 de abril de 1951, Luis Bolín, director general de turismo del gobierno de Franco recibía una carta de Luis Scatti con "los escritos de mi viaje de propaganda a Norteamérica que vd. deseaba conocer" donde también incluía sus informes (ampliamente citados en este artículo) sobre el origen de la hotelería de lujo en Madrid y un resumen de su trayectoria profesional. Scatti informaba a Bolín sobre dos temas que en ese momento preocupaban, y mucho, al director de turismo: por qué se suspendieron las gestiones con American Express que se iniciaron a principios de siglo, y en qué consistió su viaje por Europa y, sobre todo, por América. Scatti, además, aprovechó para explicarle "cómo una empresa genuinamente española pasó al control de una extranjera" ${ }^{47}$. Porque si bien a Bolín le interesaba conocer los precedentes de las relaciones turísticas con los EEUU en un momento en que él encabezaba una ambiciosa y estratégica campaña entre ambos países, Luis Scatti parecía ansioso por contar qué había pasado con el Hotel Ritz, y cómo fue la aparición de Marquet lo que determinó su suerte.

Luis Scatti es un nombre desconocido del turismo histórico español, o al menos, uno de los olvidados de la hotelería española de principios de siglo. Nació en Barcelona en 1875 y, como hijo de un trabajador de hotel, pareció encaminado desde muy joven a ese mundo en el que su padre ejerció de guía y mentor. Con 17 años salió de España para continuar su formación en Francia, Inglaterra y Alemania y recaló en Londres en 1894 donde fue discípulo del propio César Ritz que estaba en ese momento reflotando el Savoy. Pasados nueve años, en 1901, volvió a España "con todos los conocimientos para ser un buen hotelero" y encontró trabajo en Barcelona donde conoció, y fue contratado, por Arturo Vilaseca.

46 CRESPO GAllegO, Hilario: Proyecto de Congreso Hispanoamericano presentado a la Cámara Oficial de Comercio de la provincia de Madrid, 1914; Proyecto de Congreso Iberoamericano de Turismo, Madrid, Sobrinos de la Suc. de M. Minuesa de los Rios, 1926; Fiesta de la Raza. Discurso pronunciado en la sesión celebrada bajo la presidencia de S.M. el Rey Alfonso XIII, 12 de Octubre, en el Ayuntamiento de Madrid.

47 03. 49. 02. 12104 (AGA) 
Este empresario catalán, al que Scatti se referiría en su informe como el hombre interesado en abrir un hotel de lujo en Madrid, era propietario del Gran Café Colón en la nueva Plaza de Cataluña esquina con el Paseo de Gracia, un lugar inmejorable para instalar un buen hotel pero para el que necesitaba asesoramiento. Lo encontró en él, que le ayudó a convertirlo en un hotel espléndido, subiendo dos plantas de habitaciones e instalando agua caliente, calefacción central, teléfono y todas las comodidades de los grandes hoteles europeos del momento. El primer Hotel Colón era un edificio bellísimo de estilo modernista diseñado por Andreu Daudet con sesenta habitaciones, servicio de carruajes y magníficos salones acristalados que recordaban su pasado como café decimonónico. La publicidad de la época lo vendía como un hotel de primer orden y, casi con seguridad, era el mejor de la ciudad y uno de los mejores de España. Luis Scatti fue su primer director.

A pesar del éxito del hotel que, incluso en 1916 tuvo que ser ampliado, Scatti dejó Barcelona en 1906, una vez más, para trabajar fuera. Ese año recibió una interesante oferta de la compañía de vapores inglesa Elder Dempster, Co que le ofreció un contrato por cinco años y le nombró director general de sus hoteles en Jamaica (Jamaica Hotels Co. Ltd) donde estuvo hasta 1911 a cargo de cuatro hoteles donde destacaba el Constant Spring Hotel a pocos kilómetros de Kingston y uno de los más importantes de la isla, construido en 1891 para la Exposición de Jamaica. Su vuelta a España estuvo motivada por asuntos familiares, o así dejó entrever Scatti años después a Bolín y ocurrió en un momento inmejorable ya que para 1911, cuando regresó, ya funcionaba el que sería uno de los más lujosos, sino el más lujoso hotel del momento: el Ritz de Madrid. Según Scatti, fueron los propios ingleses, en concreto William Harris, presidente de la Ritz Hotels Development Company, el que le escribió, en julio de 1911, ofreciéndole el puesto que ya ocupaba desde la inauguración Antonio Mella que, según el, no estaba a la altura. La sociedad Ritz de Madrid dio el visto bueno y Scatti firmó un contrato de tres años prorrogables con el marqués de Guadalmina y Mariano de Cuadra.

En su nuevo puesto se encontró con la dificultad de conseguir la adinerada clientela que necesitaba un hotel como el Ritz, de ahí que la cuestión de la propaganda y promoción del hotel fuese una urgente necesidad. Tras una primera valoración, y basado en su propia experiencia personal, insistió en que

no estando en España debidamente organizada la propaganda, ni por lo tanto la atracción turística extranjera, especialmente la de calidad, no se podía esperar y mucho menos asegurar que los resultados fueran un éxito ${ }^{48}$

Para garantizar la necesaria clientela, España necesitaba urgentemente fomentar el turismo, darse a conocer fuera y hacer buenos contactos internacionales, siendo prioritario que el hotel tuviese en España la representación de la American Express, "la más importante del mundo inventora de los American Express travelling cheques

48 SCATTI Luis, Memoria presentada al Ritz de Madrid por Luis Scatti director del Hotel referente a su viaje de propaganda a Londres, EEUU, Alemania y París (1 de noviembre de 1912 al 4 de enero de 1913), 03. 49. 02. 12104 (AGA) 
que son aceptados en todo el mundo y muy usados por el turismo americano" y cuyo representante aquí era la francesa Credit Lyonnais ${ }^{49}$.

Así, Scatti se embarcó en un viaje promocional que le tendría fuera de España dos meses haciendo negocios, asistiendo a la inauguración de dos nuevos hoteles de su grupo (los Ritz-Carlton de Filadelfia y Montreal) pero, también de paso, dando a conocer una ciudad que ya podía ser visitada por grandes turistas que tendrían dónde alojarse. Conocedores de los planes de Scatti, desde la Asociación de Propaganda, el 16 de abril de 1912 le nombraron vocal y miembro del comité organizador del congreso de ese mismo otoño. Scatti, de hecho, espero a la finalización del congreso el 30 de octubre para viajar y por una carta de agradecimiento que le escribió Hilario Crespo a su vuelta, en febrero de 1913, sabemos que le debió enviar varios recortes y noticias de prensa con referencias a su viaje.

El informe que se conserva de su viaje ocupa unas treinta páginas detalladas de itinerarios, contactos, nombres y direcciones que nos permitirían reconstruir, en parte, el ambiente empresarial del turismo internacional de principios de siglo. Un ambiente del que interesó, sobre todo, el mundo anglosajón (sus jefes e inversores) y Alemania. La noche del día 8 de noviembre de 1912 salió de Madrid en un tren Sud-Express en dirección a París en un viaje que le llevó prácticamente un día y que apenas le retuvo una noche indispensable en la capital francesa para salir a la mañana siguiente hacia Londres, la primera gran parada de su viaje. En la capital británica estuvo alojado en el Carlton, propiedad de sus jefes ingleses y empleó varios días, desde la tarde del 3 de noviembre hasta el 8 con entrevistas y visitas donde destacó la que hizo a Errazu, uno de los socios españoles de la Sociedad Hotel Ritz, S.A. El día 8 se embarcaba rumbo a EEUU. Estuvo varios días en Nueva York y Boston porque, hasta el 26 por la mañana no tomó el Expreso para Chicago, donde llegó el 27 por la mañana. Allí se quedó hasta el 3 de diciembre cuando llegó a Washington y donde se entrevistó, entre otros con el editor del Washington Post y Juan Riaño, el embajador español. El 6 salió para Baltimore y, de allí, el 10 viajó a Filadelfia, donde estuvo apenas horas, las justas para asistir a la inauguración de un Ritz-Carlton porque, por esa misma noche tomó un tren con destino Nueva York donde estuvo dos días. El 12 de diciembre volvía a Europa pero no vía Londres sino a su nueva parada: Hamburgo donde llegó el 21 y donde se alojó en el Esplanade, otro de los grandes hoteles de Europa. El 24 al mediodía salió para Berlín donde estuvo hasta el 28 por la mañana y de allí a Frankfurt y Colonia donde pasó la noche de fin de año. El 1 de enero salió de Alemania con dirección París donde tomó el tren que le trajo de vuelta a Madrid el 3 de enero.

El viaje de Scatti fue intenso y muy profesional y, aunque tuvo mucho que ver con la gestión hotelera (estuvo muy interesado en visitar cocinas y plantas bajas y/o de fiestas además de entrevistarse con todos y cada uno de los directores de los hoteles donde se alojó) también fue de pura política turística porque parte de sus esfuerzos fueron la atracción de turismo internacional a España y la mejora de la promoción del turismo español de ahí las entrevistas con líneas trasatlánticas (Hamburg Amerika Linie, Cunard Linei....) agencias de viajes como American Express o Cook o incluso medios de comunicación y agencias de publicidad ya que pretendió que una ameri-

\footnotetext{
49 SCATTI, Luis, Memoria presentada.
} 
cana se hiciera cargo de la publicidad del turismo de Madrid. En su informe, Scatti habla de comisiones, presupuestos, cartas de recomendación, invitaciones, intercambio de tarifas o la cuestión del desembarco de coches en Gibraltar lo que indica que sus objetivos iban más allá de dar a conocer el nuevo Ritz de la ciudad. La cuestión de fondo era conseguir que los turistas vinieran a Madrid. Un objetivo que no logró y que, en los años 50 seguía preocupando al director general de turismo.

A su vuelta, Scatti empezó a ser consciente de la amenaza que se cernía sobre su hotel. En apenas seis meses el Ritz fue traspasado a Marquet y él dejó la dirección del mismo. Con él fuera,

ya no se habló más, ni nadie se preocupó tampoco en continuar las negociaciones que emprendí a resultas de mi viaje de propaganda a Norteamérica y de acuerdo con el "Fomento de turismo de Madrid" con la American Express las cuales, de haber materializado no solo hubieran sido un magnífico principio para la puesta en marcha de otras iniciativas malográndose unos sinceros propósitos puestos al servicio de la patria $^{50}$

Si aquel viaje quedó en nada, algo parecido ocurrió con él ya que, en 1914, dejó Madrid y la dirección hotelera para volver a Barcelona y dedicarse a la planificación de hoteles, balnearios, casinos y restaurantes. Entre 1917 y 1924 fue asesor técnico de la junta de gobierno del Círculo Ecuestre de Barcelona, primero en restauración y contabilidad y luego en la comisión de obras de arquitectura. En 1925 fue nombrado consejero delegado del Majestic Hotel Inglaterra S.A de Barcelona y durante la guerra se encargó de asesorar al hospital policlínico de la ciudad, primero en el departamento de contabilidad, luego, terminada la guerra, en la ampliación del edificio y reforma de las instalaciones culinarias e industriales. Ahí, en la Barcelona de principios de los años 50 se pierde la pista de uno de los españoles que más, y mejor, debió conocer el ambiente turístico internacional de la primera década del siglo.

\section{Conclusiones: 1912, más allá de Madrid}

El primer desarrollo turístico madrileño nos presenta un panorama nacional más prometedor del que habíamos pensado. El interés de grupos empresariales muy influyentes por el mercado hotelero español, viajes y contactos internacionales con objetivos estratégicos muy claros o la madurez del primer tejido turístico nacional que se reunió en el V Congreso Internacional de Turismo destierra, en parte, esa imagen de país atrasado en lo turístico, al menos, hasta los años de entreguerras. Hasta ahora, los investigadores sabíamos de la importancia del veraneo aristocrático o de los balnearios pero hay que insistir en los síntomas ya puramente turísticos (hotelería, asociacionismo, profesionalización) de una ciudad que se salía de los clásicos enclaves turísticos y que, por extensión, podría ser indicativa del turismo nacional.

La construcción de un hotel de gran lujo en Madrid en 1910 fue muy reveladora de la importancia creciente del turismo y colocaba a Madrid a la altura de las grandes

50 SCATTI, Luis, Memoria presentada. 
capitales continentales además con el sello hotelero más prestigioso del mundo, Ritz, que no pudo ocuparse personalmente del hotel madrileño pero cuyos planos fueron supervisados por su arquitecto parisino. Que, casi desde el mismo momento, el otro gran hotelero europeo se fijase en Madrid es más que indicativo. El gran lujo llegó a una ciudad en plena transformación urbanística y se ubicó en un triángulo estratégico donde confluían cultura, alta política y grandes entidades financieras por eso hay que entenderlos más allá del turismo y el viaje. Dos grandes hoteles en apenas dos años es casi un récord para una ciudad hasta entonces desatendida por la industria turística internacional pero además fue el punto de partida del interés del "mago" de la hotelería europea por nuestro país; Santander, San Sebastián y Sevilla fueron los otros escenarios de las inversiones de Marquet en España. Pero no fue solo un gran grupo internacional, entre la nómina de empresarios y financieros nacionales que empezaron a interesarse por el turismo tenemos a Urquijo, Botín, Guadalmina o los Comillas, además de la participación, aunque fuese anecdótica, del propio monarca.

Por otro lado, y en un movimiento casi contrario, de abajo a arriba y muy alejado del lujo y de las élites, el Madrid de 1912 nos demuestra que hubo un tejido turístico de base representado en una tupida red de sindicatos de iniciativa turística que eran muy activos y tenían buenas relaciones e intereses internacionales con nuestros vecinos fronterizos. El congreso que se celebró en Madrid el otoño de 1912 donde se reunieron casi trescientos congresistas de los tres países es el mejor resumen de toda una época del turismo histórico y deja ver, en sus sesiones de trabajo, las preocupaciones del sector pero también la importancia, a nivel nacional, de la activa Asociación de Propaganda de Madrid, que luchó por ser anfitriona del congreso y que tuvo en su secretario uno de los hombres clave del turismo español de principios de siglo

La aceleración del turismo madrileño también puso sobre la mesa, muy pronto, la enorme brecha entre la España turística real y la oficial. Las investigaciones más recientes sobre la historia de la administración turística española están insistiendo en ese tema que quedó muy claro precisamente en Madrid, en 191251. El congreso de turismo estuvo, en el fondo, huérfano de apoyo oficial y el comisario de turismo no se molestó ni en asistir, ni en implicarse en su organización. Cuando ese mismo otoño Luis Scatti se embarcó en su viaje internacional para dar a conocer su hotel, pero también la ciudad, estaba haciendo, probablemente, uno de los primeros viajes de política turística y contactos internacionales del turismo español. Su intensa agenda y el interés de las personas con las que se reunió contrasta con aquellos viajes a Norteamérica que hizo el responsable del turismo nacional que, hasta donde sabemos, apenas se entrevistó con millonarios para tratar asuntos personales de mecenazgo pero que poco tuvieron que ver con el desarrollo del turismo nacional. Dicho de otra manera, Luis Scatti hizo el viaje que podía, y debía, haber hecho el comisario español de turismo. El desinterés oficial por el turismo a lo mejor no cambió las cosas (la naturaleza del Estado era la que era y sus límites, muy obvios) pero deja interesantes lecturas sobre lo que la monarquía alfonsina hizo, en realidad, por la prometedora industria de los forasteros y nos da la versión definitiva sobre la Comisaría Regia del Turismo.

51 VILLAVERDE, Jorge: Sunny Spain: elaboración, negociación y difusión de un imaginario nacional español. Londres, 1914 (Tesis doctoral en curso, Instituto Universitario Europeo, Florencia). 
Y un último apunte: los hilos conductores entre el principio de siglo y el turismo de entreguerras. La primera década de siglo empezó a plantear algunos asuntos que serán muy importantes en los años 20 como fueron el americanismo y la necesidad de estrechar lazos comerciales y culturales con América pero fue más allá en el tiempo. El "rescate" que hiciera Bolín en los años 50 de la figura y el viaje de Luis Scatti también es representativa de esos asuntos que se quedaron pendientes en los primeros años del turismo español. Cuando Bolín intentó que American Express se instalara en España encontró un viejo precedente en aquel viaje de 1912 y se interesó por él. Cuarenta años después, la administración turística española se ponía el mismo reto, nunca logrado: la captación del turismo norteamericano, la mayor presencia española en los circuitos turísticos internacionales y la atracción de inversores y grandes nombres de la industria internacional. 